\title{
Profile and outcome of patients with upper gastrointestinal bleeding presenting to urban emergency departments of tertiary hospitals in Tanzania
}

\author{
Shaffin S. Rajan ${ }^{1}$, Hendry R. Sawe ${ }^{1,2^{*}}$ D, Asha J. Iyullu' ${ }^{1}$, Dereck A. Kaale ${ }^{1}$, Nancy A. Olambo ${ }^{4}$,
} Juma A. Mfinanga ${ }^{1,2}$ and Ellen J. Weber ${ }^{2,3}$

\begin{abstract}
Background: Upper gastrointestinal bleeding (UGIB) is a common emergency department (ED) presentation with high morbidity and mortality. There is a paucity of data on the profile and outcome of patients who present with UGIB to EDs, especially within limited resource settings where emergency medicine is a new specialty. We aim to describe the patient profile, clinical severity and outcomes of the patients who present with UGIB to the ED of tertiary referral hospitals in Tanzania.
\end{abstract}

Methods: This was a prospective cohort study of consecutive adult ( $\geq 18$ years) patients presenting to the EDs of Muhimbili National Hospital (ED-MNH) and MUHAS Academic Medical Centre (ED-MAMC), in Tanzania with nontraumatic upper gastrointestinal bleeding (UGIB) from July 2018 to December 2018. Patient demographic data, clinical presentation, and ED and hospital management provided were recorded. We used the clinical Rockall score to assess disease severity. The primary outcome of 7- day mortality was summarized using descriptive statistics. Regression analysis was performed to identify predictors of mortality.

Results: During the study period, 123 patients presented to one of the two EDs with an UGIB. The median age was 42 years (Interquartile range (IQR) 32-64 years), and 87 (70.7\%) were male. Hematemesis with melena was the most frequently encountered ED complaint 39 (31.7\%). Within 7 days, 23 (18.7\%) patients died and one-third 8 (34.8\%) of these died within $24 \mathrm{~h}$. There were no ED deaths. About $65.1 \%$ of the patients had severe anemia but only 60 (48.8\%) received blood transfusion in the ED. Amongst those with history of (h/o) esophageal varices $7(41.2 \%)$ did not receive octreotide. Upper Gl endoscopy, was performed on 46 (37.4\%) patients, of whom only 8 (17.4\%) received endoscopy within $24 \mathrm{~h}$ (early UGl endoscopy). All patients who received early UGl endoscopy had a low or moderate clinical Rockall score i.e. $<3$ and 3-4. No patient with scores of $>4$ received early UGl endoscopy. Age $>40$ years was a significant independent predictor of mortality $(\mathrm{OR}=7.00(95 \% \mathrm{Cl}$ 1.7-29.2). Having a high clinical Rockall score of $\geq 4$ was a significant independent predictor of mortality ( $\mathrm{OR}=6.4(95 \% \mathrm{Cl} 1.8-22.8)$.

Conclusions: In this urban ED in Sub-Saharan Africa, UGIB carried a high mortality rate. Age $>40$ years and clinical Rockall score $\geq 4$ were independent predictors of higher mortality. Future studies should focus on evaluating how to improve access to UGl endoscopy so as to improve outcomes.

Keywords: Upper gastrointestinal bleeding, Non-traumatic patients, Emergency department, Tanzania, Sub Saharan Africa.

\footnotetext{
* Correspondence: hendry_sawe@yahoo.com

'Emergency Medicine Department, Muhimbili University of Health and Allied

Science, P.O. Box 65001, Dar es Salaam, Tanzania

${ }^{2}$ Emergency Medicine Department, Muhimbili National Hospital, Dar es

Salaam, Tanzania

Full list of author information is available at the end of the article
}

(c) The Author(s). 2019 Open Access This article is distributed under the terms of the Creative Commons Attribution 4.0 International License (http://creativecommons.org/licenses/by/4.0/), which permits unrestricted use, distribution, and reproduction in any medium, provided you give appropriate credit to the original author(s) and the source, provide a link to the Creative Commons license, and indicate if changes were made. The Creative Commons Public Domain Dedication waiver (http://creativecommons.org/publicdomain/zero/1.0/) applies to the data made available in this article, unless otherwise stated. 


\section{Background}

Upper Gastrointestinal bleeding (UGIB) is a medicosurgical emergency. Although there has been a global decline in the mortality associated with UGIB, the incidence and mortality associated with GI bleeding remains high in limited income countries. In the United States, UGIB accounts for 300,000 admissions per year with approximately $5 \%$ mortality rate [1], whereas in several studies from sub-Saharan Africa, mortality ranges from 6 to $30 \%$ [2-5]. It is not clear if the higher mortality seen in low and middle income countries (LIMC's) is due to patient demographics, severity on presentation, etiology or compliance with care standards. Esophageal varices has been implicated as the most common cause of UGIB in several African studies [2-11]. Rather than being due to alcoholic liver disease as in high income countries (HIC's), varices in sub-Saharan Africa result from Schistosoma-related portal hypertension [5, 8, 12]. This is in contrast to HIC where erosive gastritis has been commonly implicated $[1,13,14]$. Other potential contributors to the higher mortality may be the severity of disease presentation, as there is little primary care in these settings, and many patients seek care very late in their disease.

Another potential contributor is failure to treat patients in accordance with management guidelines. Proton pump inhibitor (PPI) for those with suspected Nonvariceal UGIB (NVUGIB), somatostatin analogues such as octreotide and antibiotics in suspected cases of variceal UGIB (VUGIB) and in those with clinical suspicion of liver disease, timely blood transfusion and early use of endoscopy may not occur in these settings due to lack of appropriate specialists, lack of resources or supply chain issues [15-17]. A knowledge gap on the current UGIB management guidelines and recommendations amongst the health care providers may also contribute to inadequate management of UGIB cases. Prior studies in Tanzania studies demonstrate lack of appropriate care offered to patients with UGIB. Nearly half of the patients in one the studies did not receive endoscopic evaluation and treatment [5] whereas $47.1 \%$ of the patients who required blood transfusion, did not receive blood transfusion in another study [10].

There is little information on the presentation, etiology and management of patients presenting to EDs and this is particularly true for LIMCS, where emergency medicine is a new specialty. The presence of full capacity emergency departments at Muhimbili National Hospital $(\mathrm{MNH})$ and MUHAS Academic Medical Centre (MAMC) in Dar es Salaam have provided the opportunity for early stabilization and management of patients presenting with UGIB. The aim of this study was to describe the patient profile, clinical severity and outcomes of the patients who present with UGIB to the ED.

\section{Methods}

\section{Study design}

This was a prospective cohort study of adult patients presenting to two EDs in Tanzania with upper gastrointestinal bleeding from July 2018 to December 2018.

\section{Study setting}

This study was conducted at the ED- MNH and EDMAMC, Dar es Salaam Tanzania. MNH is a public, tertiary referral hospital which opened in 2010. It is the site of the first full capacity public ED in Tanzania and the primary training site for the only Emergency Medicine (EM) residency program in the country. MAMC is a recently inaugurated full capacity university health facility with a state of the art emergency medicine department, located approximately $30 \mathrm{~km}$ from $\mathrm{MNH}$. $\mathrm{MNH}$ and MAMC both have a fully equipped endoscopy unit.

\section{Study participants}

All consenting adults (age greater than or equal to 18 years) presenting with UGIB unrelated to a recent trauma were eligible for the study. We excluded patients who had previously been enrolled in the study who presented with recurrent episodes of UGIB during the period of the study.

\section{Study protocol}

Research assistants were scheduled to collect data $24 \mathrm{~h}$ a day, seven days a week and during that time patients were consecutively approached and asked for consent to be followed for the study. Demographics, clinical presentation, initial management, and ED outcomes were collected by the research assistant using information given by the patient or caregiver, the treating physician, and data found in the electronic medical record (Wellsoft ${ }^{\mathrm{rm}}$ ). A structured case report form was used to record all participants' information. All admitted patients were followed up in a hospital ward and if discharged through mobile phone calls to determine their outcome from the EMD-MNH and EMD-MAMC, at 24-h and 7-days.

\section{Assessment of disease severity}

Clinical severity was measured using the pre-endoscopic Rockall score (Table 1) and the Glasgow-Blatchford (Table 2). For pre-endoscopic Rockall score, scores $<3$ signifies low risk, scores 3-4 signifies moderate risk and score $>4$ signifies high risk for re-bleeding, mortality or surgery [19]. For Glasgow-Blatchford score, a score of $<3$ is considered Low-risk, whereas a score greater than $\geq 3$ is high risk, thus needing intervention, transfusion, endoscopy or surgery. The cut-off value for GBS severity determined as per the study by Ramfrez et al [20]. 
Table 1 Clinical (Pre-Endoscopic) Rockall Score

\begin{tabular}{lllll}
\hline COMPONENT & 0 & 1 & 2 & 3 \\
\hline AGE (yrs) & $<60$ & $60-79$ & $>80$ \\
HEMODYNAMICS & $H R<100$ & HR $>100$ & SBP $<100$ \\
& SBP $>100$ & SBP $>100$ & & \\
COMORBIDITIES & NONE & - & IHD, CHF, ANY MAJOR COMORBIDITIES & RENAL FAILURE, LIVER FAILURE, METASTASIS \\
\hline
\end{tabular}

Source: Wang2013

Total score is calculated by addition of individual scores

\section{Emergent and early upper Gl endoscopy}

Emergent upper GI endoscopy was defined as endoscopy performed within $12 \mathrm{~h}$ of ED presentation whereas early upper GI endoscopy was defined as endoscopy performed within $24 \mathrm{~h}$ of ED presentation [21].

\section{Outcomes}

The primary outcome was 7 day mortality due to any cause and not only related to GI bleeding. Secondary outcomes were ED and hospital length of stay and $24 \mathrm{~h}$ mortality.

Table 2 Glasgow-Blatchford Score

\begin{tabular}{|c|c|}
\hline ADMISSION RISK MARKER & SCORE COMPONENT \\
\hline \multicolumn{2}{|l|}{ BLOOD UREA (mmol/L) } \\
\hline $6.5-8$ & 2 \\
\hline $8.0-10.0$ & 3 \\
\hline $10.0-25.0$ & 4 \\
\hline$>25$ & 6 \\
\hline \multicolumn{2}{|l|}{ HEMOGLOBIN FOR MEN(g/dl) } \\
\hline $12.0-12.9$ & 1 \\
\hline $10.0-11.9$ & 3 \\
\hline$<10$ & 6 \\
\hline \multicolumn{2}{|l|}{ HEMOGLOBIN FOR WOMEN(g/dl) } \\
\hline $10.0-11.9$ & 1 \\
\hline$<10$ & 6 \\
\hline \multicolumn{2}{|l|}{ SYSTOLIC BLOOD PRESSURE (mmHg) } \\
\hline $100-109$ & 1 \\
\hline $90-99$ & 2 \\
\hline$<90$ & 3 \\
\hline \multicolumn{2}{|l|}{ OTHER MARKERS } \\
\hline PULSE RATE > 100 bpm & 1 \\
\hline PRESENATION WITH MELENA & 1 \\
\hline PRESENTATION WITH SYNCOPE & 2 \\
\hline HEPATIC DISEASE & 2 \\
\hline CARDIAC FAILURE & 1 \\
\hline TOTAL & \\
\hline
\end{tabular}

Source: Cheng 2012 [18]

\section{Data analysis}

Data from the case report form was entered into Research Electronic Data Capture (REDCap) software (version 7.2.2, Vanderbilt, Nashville, TN, USA) and transferred into the Statistical Package for Social Science (SPSS) (version 25.0, IBM, LTD, North Carolina, USA). Descriptive statistics were computed with continuous variables presented as mean $+/$ - standard deviation (SD) or median with it's IQR depending on distribution. Categorical variables are expressed as number and percentage. Univariate associations between categorical variables and outcomes were computed using the Pearson Chi-square test. Multivariate regression analysis was completed on variables with $p$ value $\leq 0.20$ in the univariate analysis to identify predictors of 7-day mortality due to UGIB. Statistical significance was set at $p$-value $<0.05$.

\section{Results}

During the period of study, there were 31,987 patient visits (30,800 at EMD-MNH and 1187 at EMD-MAMC). From these we identified $123(0.4 \%)$ patients with upper GI bleeding, all of whom were eligible and consented to be in the study (Fig. 1). Median age was 42 [IQR 32-64] years and $87(70.7 \%)$ were male; $87(70.7 \%)$ were married $44(35.8 \%)$ were self-employed. The majority of patients 77 (62.6\%) had been previously seen at a lower capacity health facility and transferred to one of the two study sites. Most patients $(99,80.5 \%)$ were uninsured. (Table 3$)$.

\section{Clinical presentation}

The most frequently encountered presenting complaint was a combination of hematemesis and melena, reported by 39 (31.7\%) patients. (Table 3) Nearly half of all patients (56, 45.5\%) were tachycardic on arrival. All 123 patients with UGIB were scored for the clinical Rockall score. 63 (51.2\%) of the patients had a moderate risk clinical Rockall score of 3-4. A Glasgow-Blatchford score (GBS) could be obtained for 82 patients; this was due to missing of one or more of the point of care (POC)/Lab results required for this scoring system. Of those scored, the majority $(71,86.6 \%)$ had GBS score of $\geq 3$, thus characterizing these patients as high risk for adverse events. Hemoglobin $(\mathrm{Hb})$ count was obtained in 106 patients. $69(65.09 \%)$ of these patients had a $\mathrm{Hb}<8 \mathrm{~g} / \mathrm{dl}$, thus categorizing them as severe anemia according to the World Health Organization (WHO) classification. 


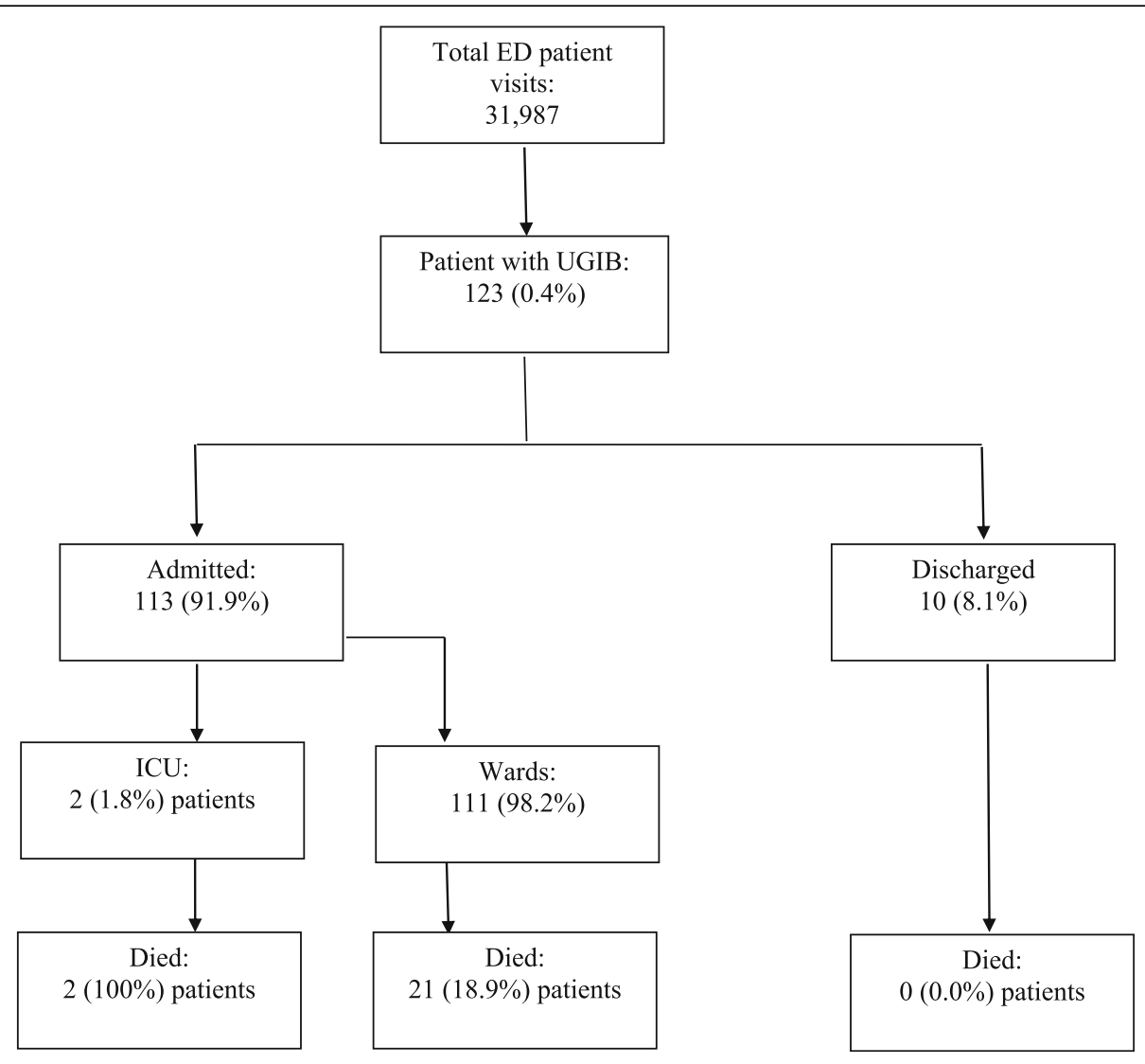

Fig. 1 STROBE Flow Diagram

Management strategies at the emergency department

The majority of the patients $(71.5 \%)$ received intravenous fluid whilst at the ED (Mean volume: $1394.3 \mathrm{mls}$ ). Proton pump inhibitor (PPI) was given in $71.5 \%$ of patients, of which $38.6 \%$ had prior peptic ulcer disease (PUD) history. 19 (59.4\%) of those with a high clinical Rockall score, i.e. $\geq 4$ received high dose PPI (i.e. $80 \mathrm{mg}$ ) whereas $12(37.5 \%)$ of those with the high clinical Rockall score did not receive any PPI. A small proportion of patients (18.7 and 20.3\%) received Octreotide and Tranexamic acid respectively. Less than half (10, 43.5\%) of those receiving Octreotide had prior h/o esophageal varices. Amongst those with h/o esophageal varices (7, $41.2 \%)$ did not receive octreotide. A minority (8, 18.6\%) of those with a known history of liver disease received antibiotics in the ED. Nearly half $(48.8 \%)$ of patients received blood transfusion, with $80 \%$ of them receiving 1 unit of blood. No patient received emergent endoscopy.

\section{Financial status and ED management (including UGI endoscopy)}

We found no statistical significant between the financial status i.e. insured or non-insured patients and the ED management provided nor with the UGI endoscopy provision. (Table 4).

\section{Hospital management}

Upper GI endoscopy was performed in 46 (37.4\%) of patients. Of these, $8(17.4 \%)$ received early Upper GI endoscopy within $24 \mathrm{~h}$. All patients who received early UGI endoscopy had a low or moderate clinical Rockall score i.e. $<3$ and 3-4. No patient with scores of $>4$ received early UGI endoscopy. This may imply that the clinical Rockall score in this study was more of a predictor to who receives UGI endoscopy, rather than a predictor of mortality. The majority of patients received UGI endoscopy from $72 \mathrm{~h}$ up to 7 days post admission 15 (32.6\%). None of the patients with h/o esophageal varices received early endoscopy (Table 5).

\section{Disposition and follow up}

Of those enrolled, 113 (91.8\%) were admitted and 10 were discharged or eloped prior to discharge. All patients were followed up, including those discharged. Amongst those admitted, 111 (90.2\%) were admitted to the wards and $2(1.6 \%)$ to the intensive care unit (ICU). The overall 7 -day mortality was $18.7 \%$. Both patients 
Table 3 Demographic and Clinical Characteristics of adult patients presenting with UGIB

\begin{tabular}{|c|c|}
\hline VARIABLE & FREQUENCY (\%) \\
\hline \multicolumn{2}{|l|}{ Age (yrs.) } \\
\hline Median age & 42 years (IQR 32-64) \\
\hline$>40$ years & $64(52.0 \%)$ \\
\hline \multicolumn{2}{|l|}{ Sex } \\
\hline Male & $87(70.7 \%)$ \\
\hline \multicolumn{2}{|l|}{ Referral Status } \\
\hline Hospital referred & $77(62.6 \%)$ \\
\hline \multicolumn{2}{|l|}{ Insurance Status } \\
\hline Uninsured & $99(80.5 \%)$ \\
\hline \multicolumn{2}{|l|}{ Presenting Complaint } \\
\hline Hematemesis & $34(27.6 \%)$ \\
\hline Melena & $33(26.8 \%)$ \\
\hline Hematochezia & $11(8.9 \%)$ \\
\hline Hematemesis and Melena & $39(31.7 \%)$ \\
\hline Hematemesis and Hematochezia & $6(4.9 \%)$ \\
\hline Active Bleeding episode in ED & $8(6.5 \%)$ \\
\hline \multicolumn{2}{|l|}{ Vital Signs } \\
\hline Tachycardia (HR > 100 /min) & $56(45.5 \%)$ \\
\hline Hypotension (SBP < 100 mmHg) & $23(18.7 \%)$ \\
\hline Sp02<90\% & $6(4.89 \%)$ \\
\hline \multicolumn{2}{|l|}{ Medical history } \\
\hline PUD & $39(40.2 \%)$ \\
\hline UGIB & $25(25.8 \%)$ \\
\hline Esophageal Varices & $17(17.5 \%)$ \\
\hline Liver disease & $12(10.3 \%)$ \\
\hline Others & $51(52.6 \%)$ \\
\hline UGl endoscopy & $20(16.3 \%)$ \\
\hline Band ligation & $4(3.3 \%)$ \\
\hline \multicolumn{2}{|l|}{ Clinical Rockall Score $(n=123)$} \\
\hline $3-4$ & $63(51.2 \%)$ \\
\hline$<3$ & $52(42.3 \%)$ \\
\hline$>4$ & $8(6.5 \%)$ \\
\hline \multicolumn{2}{|l|}{ Glasgow-Blatchford Score $(n=82)^{*}$} \\
\hline$\geq 3$ & $71(86.6 \%)$ \\
\hline$<3$ & 11 (13.4\%) \\
\hline
\end{tabular}

${ }^{*}$ Cut-off value for GBS severity determined as per the study by Ramfrez et al. [20]

admitted to the ICU died within 7 days. Among those admitted to the ward, 21 (18.9\%) patients died within 7 days and no patients who were discharged died. 24-h mortality was 8 (34.8\%).

\section{Predictors of 7-day mortality}

Factors significantly associated with mortality in univariate analysis were age group $>40$, prior $\mathrm{H} / \mathrm{o}$ liver disease,
Table 4 Cross-tabulation Analysis between Financial Status and management provided

\begin{tabular}{llll}
\hline & \multicolumn{2}{l}{ INSURED NON-INSURED } & $p$-value \\
& $n=24 n=99$ & \\
\hline ED Management & & & \\
Intravenous fluid & $18(75.0 \%)$ & $70(70.7 \%)$ & 0.676 \\
Octreotide & $4(16.7 \%)$ & $19(19.2 \%)$ & 0.776 \\
Tranexamic acid & $2(8.3 \%)$ & $23(23.2 \%)$ & 0.104 \\
PPI & $15(62.5 \%)$ & $73(73.7 \%)$ & 0.274 \\
Antibiotics & $12(50.0 \%)$ & $31(31.3 \%)$ & 0.085 \\
Blood Transfusion & $9(37.5 \%)$ & $51(51.5 \%)$ & 0.218 \\
Receiving Endoscopy & $9(37.5 \%)$ & $37(37.4 \%)$ & 0.991 \\
\hline
\end{tabular}

active bleeding episode in the ED, provision of antibiotics in the ED and clinical Rockall score $\geq 4$ (Table 6).

In multivariate regression, age and endoscopy were independently associated with 7 day outcome: age more than 40 years was independently associated with increased mortality, whereas receiving endoscopy was associated with a reduced the risk of mortality. (Table 7).

\section{Discussion}

UGIB in this limited income setting was associated with a high mortality rate $(18.7 \%)$. One third of all deaths occurred in the first $24 \mathrm{~h}$. This may be a reflection of the

Table 5 Management Strategies at Emergency Department

\begin{tabular}{ll}
\hline VARIABLES & FREQUENCY (\%) \\
\hline ED Management & \\
Intravenous fluid & $88(71.5 \%)$ \\
Proton pump Inhibitor & $88(71.5 \%)$ \\
Blood Transfusion (BT) & $60(48.8 \%)$ \\
Antibiotics & $43(35.0 \%)$ \\
Tranexamic acid & $25(20.3 \%)$ \\
Octreotide & $23(18.7 \%)$ \\
Inotropy $/$ asopressor support & $3(2.4 \%)$ \\
NGT Placement & $3(2.4 \%)$ \\
Emergent endoscopy & $0(0.0 \%)$ \\
POC results & $n(n / N)$ \\
Hemoglobin $<8 \mathrm{~g} / \mathrm{dl}(n=106)^{*}$ & $69(65.09 \%)$ \\
Urea $>7.1$ mmol/I $(n=85)^{* *}$ & $51(60.0 \%)$ \\
Lactate $>2$ meq/I $(n=20)$ & $11(55.0 \%)$ \\
UGl Endoscopy & $46(37.4 \%)$ \\
Time to Endoscopy & \\
$>72 \mathrm{~h}$ & $15(32.6 \%)$ \\
$24-48 \mathrm{~h}$ & $13(28.3 \%)$ \\
$48-72 \mathrm{~h}$ & $10(21.7 \%)$ \\
Within $24 \mathrm{~h}$ & $8(17.4 \%)$ \\
\hline
\end{tabular}

*Cut-off value based on WHO Anemia severity classification ${ }^{*}$ Cut-off based on the high normal limit as stated in Glasgow-Blatchford score 
Table 6 Univariate Analysis of Factors Associated With of 7-Day Mortality

\begin{tabular}{|c|c|c|c|}
\hline & \multicolumn{2}{|c|}{$\begin{array}{l}\text { DIED SURVIVED } \\
n=23 n=100\end{array}$} & $\begin{array}{l}p \text { - value } \\
0.005\end{array}$ \\
\hline$\leq 40$ & $5(21.7 \%)$ & $54(54.0 \%)$ & \\
\hline$>40$ & $18(78.3 \%)$ & $46(46.0 \%)$ & \\
\hline Male Sex & $14(60.9 \%)$ & $73(73.0 \%)$ & 0.249 \\
\hline \multicolumn{4}{|l|}{ Medical History } \\
\hline UGIB & $5(21.7 \%)$ & $20(20.0 \%)$ & 0.852 \\
\hline PUD & $7(30.4 \%)$ & $32(32.0 \%)$ & 0.884 \\
\hline Liver Disease & $6(26.1 \%)$ & $6(6.0 \%)$ & 0.003 \\
\hline Esophageal Varices & $3(13.0 \%)$ & $14(14.0 \%)$ & 0.905 \\
\hline UGI Endoscopy & $3(13.0 \%)$ & $17(17.0 \%)$ & 0.643 \\
\hline Prior Hospitalization & $18(78.3 \%)$ & $70(70.0 \%)$ & 0.429 \\
\hline Active Bleeding Episode In ED & $5(21.7 \%)$ & $3(30.0 \%)$ & 0.001 \\
\hline \multicolumn{4}{|l|}{ ED Management } \\
\hline Intravenous fluid & $19(82.6 \%)$ & $69(69.0 \%)$ & 0.192 \\
\hline Octreotide & $6(26.1 \%)$ & $17(17.0 \%)$ & 0.314 \\
\hline Tranexamic acid & $6(26.1 \%)$ & 19 (19.0\%) & 0.446 \\
\hline PPI & $15(65.2 \%)$ & $73(73.0 \%)$ & 0.456 \\
\hline Antibiotics & $13(56.5 \%)$ & $30(30.0 \%)$ & 0.016 \\
\hline Blood Transfusion & $13(56.5 \%)$ & $46(46.0 \%)$ & 0.410 \\
\hline Receiving Endoscopy & $4(17.4 \%)$ & $42(42.0 \%)$ & 0.028 \\
\hline Clinical Rockall Score & & & $<0.001$ \\
\hline$<4$ & $8(34.8 \%)$ & 83 (83.0\%) & \\
\hline$\geq 4$ & 15 (65.2\%) & 17 (17.0\%) & \\
\hline
\end{tabular}

high disease severity seen among these patients, but also could reflect that patients did not receive guidelineadherent care. In particular, while endoscopic evaluation at any point during the hospital stay was found to be protective factor against mortality, only $37.4 \%$ of the patients received UGI endoscopy and only a handful

Table 7 Multivariate Regression of Predictors of Mortality

\begin{tabular}{lll}
\hline & OR $(95 \%$ Cl) & p-value \\
\hline Age $>$ 40 years & $7.0(1.7-29.2)$ & 0.007 \\
H/O Liver Disease & $0.949(0.2-4.8)$ & 0.949 \\
Active Bleeding Episode in Ed & $3.4(0.6-21.1)$ & 0.185 \\
Intravenous Fluid & $1.7(0.4-7.7)$ & 0.475 \\
Antibiotics In ED & $3.8(0.9-15.2)$ & 0.055 \\
Endoscopy & $0.4(0.1-1.6)$ & 0.198 \\
Clinical Rockall Score $\geq 4^{*}$ & $6.4(1.8-22.8)$ & 0.005 \\
\hline
\end{tabular}

*Cut-off value for clinical Rockall score as per study by Wang et al. [19] Variables included above are those with $p$ value $<0.02$ in the univariate analysis received early UGI endoscopy i.e. within $24 \mathrm{~h}$ of ED presentation.

Compared with studies from HIC, patients in our study were of younger age, with the median age being 42 years [1, 13, 14, 22]. We observed that age above 40 years was an independent predictor of mortality. This has also been noted in prior studies both in $\mathrm{HIC}$ and LMIC [1, 5, 13, 14].

The mortality observed in our study is much higher compared to developed countries [1, 13, 14]. Factors contributing to the high observed mortality rate include; higher disease severity, disposition status and gaps in management of these patients.

Our patients had a higher disease severity in comparison to those in HIC. A large proportion of our patients had a moderate to severe clinical Rockall score (i.e. score of 3-4 and >4) as compared to a Chinese study carried out in the ED, where most (50.6\%) had a low Rockall score of 0-2 [23]. Clinical Rockall score $\geq 4$ was found to be an independent predictor of higher mortality. This was also observed in several other studies (18, 22). Similarly, the Glasgow-Blatchford among patients in our study was much higher compared to a British study carried out between the years 2008-2009, where the majority of patients had a GBS score $\leq 2$ [24]. Severe anemia $(\mathrm{Hb}<8 \mathrm{~g} / \mathrm{dl})$ was observed in over a half of our patients while in studies conducted in HIC, less than one-third of the patients had a $\mathrm{Hb}$ count suggestive of severe anemia [13, 14, 25].

As part of the ED management, majority of the patients received intravenous fluids and proton-pump inhibitor (PPI). Although international guidelines such as the European Society of Gastrointestinal Endoscopy Guidelines (ESGE) suggest use of high dose PPI followed by an hourly infusion, 19 (59.4\%) patients with a high clinical Rockall score of $\geq 4$ received an initial high dose PPI. It is worthy to note that none of the patients received the hourly PPI infusion as recommended in the guidelines. A knowledge gap concerning the current recommendations amongst the medical care providers maybe the plausible to this shortcoming. As per the departmental protocol, PPI should be given to all patients presenting with UGIB. This may reason out to why PPI was the first choice of drug prior to undergoing endoscopy. A small proportion of the patients received octreotide, although only half of them had indication for its use based on historical features (such esophageal varices and liver diseases) and many of those with such a history did not receive this medication. The utilization was probably limited by the availability and the cost of the drug in our setting. But the fact that some received it inappropriately suggests a knowledge gap. Antibiotic administration especially in patients with prior history of liver disease and esophageal varices was low. The 
National Institute of Health and Care Excellence (NICE) in the UK, emphasizes prophylactic antibiotic use in patients with suspected or confirmed variceal bleed or a history suggestive of liver disease including cirrhosis [15]. Provision of antibiotics was associated with increased incidence of mortality only in univariate analysis, probably due to confounding with the presence of liver disease which is associated with a poor outcome. Finally, despite the high proportion of patients with severe anemia, less than half of the patients received transfusion. A previous study carried out at $\mathrm{MNH}$, revealed difficulties in obtaining blood for transfusion, including a general unavailability of blood, scarcity of un-cross matched blood at the blood bank along with delays in obtaining blood [26]. It is worth noting that ED management of UGIB is guided by the presence of departmental protocol for various conditions including UGIB. As such, provision of certain management may be guided by the protocol.

Only a small proportion of patients were admitted to ICU. In view of the higher disease severity in our study, a larger number of patients would be expected to be admitted into the ICU. Admission to the general ward implies that these patients were unlikely to receive the necessary aggressive care for their severity of illness; thus contributing to a high mortality rate observed. In a systematic review by Chiu et al it was noted that the majority of patients with a higher severity score were admitted to the ICU [27]. ICU admission for such high-risk patients has further been recommended in couple of international guidelines $[15,16]$. Although the reasons for low levels of ICU admission was not determined in this study, it is presumed to be due to the limited number of ICU beds compared with the patient volume and acuity that is seen at these national referral hospitals.

UGI Endoscopic evaluation and treatment is a major cornerstone in the management of UGIB and early UGI endoscopy is associated with reduced mortality and hospital length of stay [28, 29]. Just over a third of patients (37.4\%) in our study received UGI endoscopy at any time during the hospital stay; moreover only $17.5 \%$ received early endoscopy (within $24 \mathrm{~h}$ ) and none received emergent endoscopy. The commonly used methods of hemostatic control of bleeders in our endoscopy units were hemoclipping and argon plasma coagulation (APC). Esophageal banding is usually reserved for variceal bleeders in our study setting. It is also worth noting that esophageal binding kits are not readily available in the hospital stores. Studies in HIC show that a larger proportion of their patients receive UGI endoscopy, with most receiving early UGI endoscopies $[13,14]$. While literature suggests endoscopy should be done within $24 \mathrm{~h}$, we found that endoscopy at any time was associated with lower mortality. This finding was statically significant in the univariate analysis but not in the multivariate regression analysis. This may be observed due to the fact that the study was not powered to evaluate the impact of endoscopy to mortality. Further studies with a larger sample size may be useful to evaluate the impact of UGI endoscopy on mortality.

\section{Limitations}

This study was conducted at two high capacity, referral EDs and so the patient population and outcomes could be different at smaller, or lower capacity facilities. However, because these EDs receive referrals from all over the country, the patients sampled likely provide a wide representation of the Tanzanian population.

Investigations were ordered at the discretion of the physician, and thus not all patients received all tests. Some laboratory variables were not obtained for all the patients, thus this may have underestimated or overestimated the significance of these variables to the outcomes under study, including the scores such as GlasgowBlatchford score.

The sample size for the study was estimated for our overall outcome of mortality, but not necessarily for risk factors analysis, and thus some factors not found to be significant may show statistical significance in a larger study.

\section{Conclusion}

The mortality rate for UGIB in our setting remains substantially higher than in non-African countries. This appears to be due to the higher severity of disease in our patients, lack of ICU care, and inadequate adherence to treatment guidelines for medication and endoscopy. Some efforts may have been met with lack of resources, however further studies are needed to assess the knowledge of providers on managing patients with UGIB and familiarity with the international recommendations. Endoscopic evaluation and treatment at any point during the hospital stay still remains an independent predictor of mortality. Efforts are needed to increase the number of patients receiving endoscopic evaluation and treatment in a timely fashion in LIMC.

\section{Abbreviations}

BP: Blood Pressure; ED: Emergency Department; EMD: Emergency Medicine Department; GBS: Glasgow-Blatchford score; HB: Hemoglobin; HIC: High

Income Countries; HR: Heart rate; HVPG: Hepatic Venous Pressure Gradient; MAMC: MUHAS Academic Medical Center; MNH: Muhimbili National Hospital; MUHAS: Muhimbili University of Health and Allied Science; NVUGIB: NonVariceal Upper Gastrointestinal Bleeding; POC: Point of Care investigations; PPI: Proton Pump Inhibitor; PUB: Peptic Ulcer Bleed; RS: Rockall score; SBP: Systolic blood pressure; UGIB: Upper Gastrointestinal Bleeding; VUGIB: Variceal Upper Gastrointestinal bleeding

\section{Acknowledgements}

The author would like to thank all the study participants, Dr. Paulina Nkondora, and research assistants for making this project a success. 


\section{Availability of data and material}

The dataset supporting the conclusion of this article is available from the authors on request.

\section{Consent to publish}

Not applicable.

\section{Authors' contributions}

SSR was involved in the study design conceptualization, data collection, analysis and interpretation, drafted the manuscript, and made all necessary changes to the manuscript. HRS involved in the study design conceptualization, review of the data analysis and interpretation, and critical review of the manuscript. AJl was involved in the study design conceptualization, data analysis and interpretation and revision of the manuscript. DAK was involved in the study design, data analysis and interpretation and revision of the manuscript. JAM was involved in the study design, data analysis and interpretation, and revision of the manuscript. EJW was involved in the study design conceptualization, review of the data analysis and interpretation together with revision of the manuscript. NAO was involved in the study design, data analysis and interpretation and revision of the manuscript. All authors have read and approved the final manuscript.

\section{Funding}

This was a non-funded project; the principal investigators used their own funds to support the data collection and logistics.

\section{Ethics approval and consent to participate}

The study was conducted after obtaining permision from the MUHAS Institutional Review Board and MNH. All patients were entered into the study after an informed consent was signed either by the patients themselves or their guardians in the case where patients were not able to do so. The study received the waiver of consent from the MUHAS Institutional Review Board in case patients weren't able to consent such as unconscious patients with absence of relatives to provide consent. Confidendiality was observed in handling the information collected on the Case Report Form. The data obtained during the study was kept anonymous. All patients received treatment as per standard hospital policies.

\section{Competing interests}

The author declare no conflicts of interest.

\section{Author details}

'Emergency Medicine Department, Muhimbili University of Health and Allied Science, P.O. Box 65001, Dar es Salaam, Tanzania. ${ }^{2}$ Emergency Medicine Department, Muhimbili National Hospital, Dar es Salaam, Tanzania. ${ }^{3}$ Department of Emergency Medicine, University of California, San Francisco, CA, USA. ${ }^{4}$ Critical Care Unit, Regency Medical Centre, Dar es Salaam, Tanzania.

Received: 26 July 2019 Accepted: 28 November 2019 Published online: 10 December 2019

\section{References}

1. Tielleman T, Bujanda D, Cryer B. Epidemiology and risk factors for upper gastrointestinal bleeding. Gastrointest Endosc Clin N Am [Internet]. 2015; 25(3):415-28. https://doi.org/10.1016/j.giec.2015.02.010.

2. Mulima G, Qureshi JS, Shores C, Tamimi S, Klackenberg H, Andrén-Sandberg $\AA$. Upper gastrointestinal bleeding at a public referal hospital in Malawi. Surg Sci Up Gastrointest Bleeding Public Ref Hosp Malawi Surg Sci [Internet]. 2010;5(5):501-7. Available from: https://www.scirp.org/pdf/SS_2 014111715411394.pdf.

3. Elsebaey MA, Elashry H, Elbedewy TA, Elhadidy AA, Esheba NE, Ezat S, et al. Predictors of in-hospital mortality in a cohort of elderly Egyptian patients with acute upper gastrointestinal bleeding. Medicine. 2018;16(December 2017):1-5.

4. Alema ON, Martin DO, Okello TR. Endoscopic findings in upper gastrointestinal bleeding patients at Lacor hospital, northern Uganda. Afr Health Sci. 2012;12(4):518-21.
5. Moledina SM, Komba E. Risk factors for mortality among patients admitted with upper gastrointestinal bleeding at a tertiary hospital: a prospective cohort study. BMC Gastroenterol. 2017;17(1):1-11.

6. Kimu N. The profile of patients with acute upper gastrointestinal tract bleeding as seen at Muhimbili National Hospital: Muhimbili University of Health and Allied Sciences; 2007. http://dspace.muhas.ac.tz:8080/xmlui/ handle/123456789/1140

7. Rukewe A, Otegbayo JA, Fatiregun A, Statistics M. Clinical characteristics and outcome of patients with upper gastrointestinal bleeding at the emergency department of a tertiary hospital in Nigeria. Ann Ibadan Postgrad Med. 2015;13(2):89-93.

8. Chofle AA, Jaka H, Koy M, Smart LR, Kabangila R, Ewings FM, et al. Oesophageal varices, schistosomiasis, and mortality among patients admitted with haematemesis in Mwanza, Tanzania: a prospective cohort study. BMC Infect Dis. 2014;14(1):1-10.

9. Mwanahawa S, Segni Mekonnen A, Charles M, Gibson K. The aetiology, management and clinical outcome of upper gastrointestinal bleeding among patients admitted at the Kilimanjaro Christian Medical Centre in Moshi, Tanzania. Tanzania J Heal Res. 2010;12(4):286.

10. Jaka H, Koy M, Liwa A, Kabangila R, Mirambo M, Scheppach W, et al. A fibreoptic endoscopic study of upper gastrointestinal bleeding at Bugando Medical Centre in northwestern Tanzania: a retrospective review of 240 cases. BMC Res Notes [Internet]. 2012;5(1):200. Available from: http:// bmcresnotes.biomedcentral.com/articles/10.1186/1756-0500-5-200

11. Alatise OI, Aderibigbe AS, Adisa AO, Adekanle O, Agbakwuru AE, Arigbabu AO. Management of overt upper gastrointestinal bleeding in a low resource setting: a real world report from Nigeria. BMC Gastroenterol. 2014;14(1):1-9.

12. Opio CK, Kazibwe F, Ocama P, Rejani L, Belousova EN, Ajal P. Profiling lifetime episodes of upper gastrointestinal bleeding among patients from rural sub-Saharan Africa where schistosoma mansoni is endemic. Pan Afr Med J. 2016;24:1-9.

13. Thiebaud P, Yordanov Y, Galimard J, Raynal P, Beaune S, Jacquin L, et al. Management of upper gastrointestinal bleeding in emergency departments, from bleeding symptoms to diagnosis: a prospective, multicenter, observational study. Scand J Trauma Resusc Emerg Med. 2017;25(78):1-9.

14. Hreinsson JP, Kalaitzakis E, Gudmundsson S, Björnsson ES. Upper gastrointestinal bleeding: incidence, etiology and outcomes in a populationbased setting. Scand J Gastroenterol. 2013;(December 2012):439-47.

15. Dworzynski K, Pollit V, Kelsey A, Higgins B, Palmer K. Management of acute upper gastrointestinal bleeding: summary of NICE guidance. BMJ [Internet]. 2012;344:e3412-2. Available from: http://www.bmj.com/cgi/doi/10.1136/ bmj.e3412

16. Barkun AN, Bardou M, Kuipers EJ, Sung J, Hunt RH. Annals of internal medicine clinical guidelines international consensus recommendations on the management of patients with nonvariceal upper gastrointestinal bleeding. Ann Intern Med. 2010;152:101-13.

17. Rotondano G, Hucl T, Dinis-ribeiro M, Marmo R, Racz I, Arezzo A. Diagnosis and management of nonvariceal upper gastrointestinal hemorrhage: European Society of Gastrointestinal Endoscopy (ESGE) Guideline Authors European Society of Gastrointestinal Endoscopy. Eur Soc Gastrointest Endosc Guidel. 2015:47:1-46.

18. Cheng DW, Lu YW, Teller T, Sekhon HK, Wu BU. A modified Glasgow Blatchford Score improves risk stratification in upper gastrointestinal bleed: a prospective comparison of scoring systems. Aliment Pharmacol Ther. 2012:36(8):782-9.

19. Wang C, Qin J, Wang J, Sun C, Cao T, Zhu D. Rockall score in predicting outcomes of elderly patients with acute upper gastrointestinal bleeding. World J Gastroenterol. 2013;19(22):3466-72.

20. Recio-Ramírez JM, Sánchez-Sánchez M d P, Peña-Ojeda JA, FernándezRomero E, Aguilera-Peña M, del-Campo-Molina E, et al. The predictive capacity of the Glasgow-Blatchford score for the risk stratification of upper gastrointestinal bleeding in an emergency department. Rev Esp Enfermedades Dig. 2015;107(5):262-7.

21. Saltzman J. Approach to acute upper gastrointestinal bleeding in adults - UpToDate [Internet]. 2019 [cited 2019 Apr 26]. Available from: https:/mww.uptodate.com/ contents/approach-to-acute-upper-gastrointestinal-bleeding-in-adults\#H1

22. Witting MD, Magder L, Heins AE, Mattu A, Granja CA, Baumgarten M. ED predictors of upper gastrointestinal tract bleeding in patients without hematemesis. Am J Emerg Med. 2006;24(3):280-5.

23. Tang Y, Shen J, Zhang F, Zhou X, Tang Z, You T. American Journal of Emergency Medicine Scoring systems used to predict mortality in patients 
with acute upper gastrointestinal bleeding in the ED. Am J Emerg Med [Internet]. 2018;36(1):27-32. https://doi.org/10.1016/j.ajem.2017.06.053.

24. Le Jeune IR, Gordon AL, Farrugia D, Manwani R, Guha IN, James MW. Safe discharge of patients with low-risk upper gastrointestinal bleeding (UGIB): can the use of Glasgow-Blatchford Bleeding Score be extended? Acute Med. 2011;10(4):176-81.

25. Do SEB, Chudnofsky CR, Sorondo B, Dominici P, Do SJP. Incidence of occult upper gastrointestinal bleeding in patients presenting to the ED with hematochezia. Am J Emerg Med. 2007:340-4.

26. Shari CR, Sawe HR, Murray BL, Mwafongo VG, Mfinanga JA, Runyon MS. Emergency blood transfusion practices among anaemic children presenting to an urban emergency department of a tertiary hospital in Tanzania. BMC Hematol. 2017;17(19):1-8.

27. Chiu PWY, Ng EKW. Predicting poor outcome from acute upper gastrointestinal hemorrhage. Gastroenterol Clin North Am [Internet]. 2009; 38(2):215-30. https://doi.org/10.1016/j.gtc.2009.03.009.

28. Cho SH, Lee YS, Kim YJ, Sohn CH, Ahn S, Seo DW, et al. Outcomes and role of urgent endoscopy in high-risk patients with acute nonvariceal gastrointestinal bleeding. Clin Gastroenterol Hepatol [Internet]. 2017;16(3): 370-7. https://doi.org/10.1016/..cgh.2017.06.029.

29. Sarin N, Monga N, Adams PC. Time to endoscopy and outcomes in upper gastrointestinal bleeding. Can J Gastroenterol. 2009;23(7):489-93.

\section{Publisher's Note}

Springer Nature remains neutral with regard to jurisdictional claims in published maps and institutional affiliations.

Ready to submit your research? Choose BMC and benefit from:

- fast, convenient online submission

- thorough peer review by experienced researchers in your field

- rapid publication on acceptance

- support for research data, including large and complex data types

- gold Open Access which fosters wider collaboration and increased citations

- maximum visibility for your research: over $100 \mathrm{M}$ website views per year

At BMC, research is always in progress.

Learn more biomedcentral.com/submissions 